\title{
Análise geométrica de um canal aletado com transferência de calor por convecção mista através do Design Construtal
}

\author{
Geometric analysis of a finned channel with heat transfer by mixed convection and employing \\ Constructal Design
}

\author{
M. V. Altnetter ${ }^{1 *}$; M. S. Pereira ${ }^{2}$; L. A. Isoldi ${ }^{1,2}$; L. A. O. Rocha ${ }^{3}$; B. D. Amaral \\ Rodriguez $^{1}$; E. D. dos Santos ${ }^{1,2}$ \\ ${ }^{1}$ Programa de Pós-Graduação em Modelagem Computacional, Universidade Federal do Rio Grande (FURG), 96201 - \\ 900, Rio Grande-RS, Brasil. \\ ${ }^{2}$ Programa de Pós-Graduação em Engenharia Oceânica, Universidade Federal do Rio Grande (FURG), 96201-900, \\ Rio Grande-RS, Brasil. \\ ${ }^{3}$ Departamento de Engenharia Mecânica, Universidade Federal do Rio Grande do Sul, Rua Sarmento Leite, 425, \\ 90050-170, Porto Alegre-RS, Brasil.
}

*maiconunipampa@gmail.com

(Recebido em 12 de abril de 2017; aceito em 22 de maio de 2017)

\begin{abstract}
$\mathrm{O}$ presente estudo numérico consiste em avaliar a geometria de um canal horizontal com duas aletas retangulares inseridas nas superfícies submetido a escoamentos bidimensionais, permanentes, laminares, incompressíveis e com convecção mista. A geometria varia de acordo com o método Design Construtal. As simulações foram realizadas com o fluido sendo o ar, com números de Prandtl e Reynolds constantes e iguais a $\operatorname{Pr}=0,71$ e $R e_{\mathrm{H}}=100$ e para três diferentes números de Grashof, $G r_{\mathrm{H}}=10^{3}, 10^{4}$ e $10^{5}$. Foram solucionadas as equações de conservação de massa, de movimento e energia com uso do Método de Volumes Finitos. O principal objetivo é avaliar a influência da geometria das aletas retangulares inseridas no canal, bem como a distância entre elas, sobre a taxa de transferência de calor. No problema a área do canal e a área de ocupação da aleta em relação ao canal são as restrições geométricas do problema. Além disso, são avaliados três graus de liberdade: $H_{1} / L_{1}$ (razão entre altura e largura da aleta posicionada a montante no escoamento), $H_{2} / L_{2}$ (razão entre altura e largura da aleta posicionada a jusante no escoamento) e $L_{3}$ (distância entre centros das aletas). Com essa análise realizada, o método Design Construtal permitiu uma melhoria significativa no desempenho térmico dos sistemas aletados, com uma diferença entre os mínimos e máximos globais de mais de $100 \%$ quando comparados aos mesmos parâmetros de escoamento. Os resultados mostraram a importância de realizar uma análise geométrica do problema proposto para melhorar o desempenho térmico do sistema.
\end{abstract}

Palavras-chave: Design Construtal, Aletas Retangulares, Convecção Mista

The present numerical study consists in evaluating the geometry of a horizontal channel with two rectangular fins inserted in the surfaces of the channel submitted to two-dimensional, steady, laminar, incompressible flows with mixed convective heat transfer. The geometry varies according to the Constructal Design method. The simulations were performed with air working fluid, with Prandtl and Reynolds numbers constant and equal to $P r=0.71$ and $R e_{\mathrm{H}}=100$ and for three different Grashof numbers, $G r_{\mathrm{H}}=10^{3}, 10^{4}$ and $10^{5}$. The conservation equations of mass, momentum and energy were approximatelly solved with the Finite Volume Method. The main objective here is to evaluate the influence of the geometry of the rectangular fins inserted in the channel, as well as, the distance between them, on the heat transfer rate. In this problem the channel area and the area of occupation of the fin relative to the channel are the geometric constraints of the problem. In addition, three degrees of freedom are evaluated: $H_{1} / L_{1}$ (ratio between height and width of the upstream fin), $H_{2} / L_{2}$ (ratio between height and width of the downstream fin) and $L_{3}$ (distance between the fins center). With this analysis, the Constructal Design method allowed a significant improvement in the thermal performance of the finite systems, with a difference between the global minimums and maximums of more than $100 \%$ when compared within the same flow parameters. Results showed the importance to realize a geometric evaluation of the proponed problem to improve the thermal performance of the system. 


\section{INTRODUÇÃO}

O estudo sobre o mecanismo de transferência de calor por convecção apresenta grande relevância no meio científico devido à possibilidade de inúmeras aplicações na Engenharia. Tais aplicações podem ser vistas em alguns problemas como: projeto, operação e manutenção de trocadores de calor, que são encontrados em centrais térmicas, instalações de aquecimento de água, refrigeração de radiadores de veículos automotores; isolamentos térmicos para a minimização da transferência de calor em equipamentos térmicos, câmaras frias e para conforto térmico em ambientes de trabalho e residencial; controle de temperatura em equipamentos que operam em faixas específicas ou com um valor crítico, como componentes de computadores $[1,2]$.

A proposta do presente trabalho assemelha-se a configuração geométrica de um trocador de calor tubo casco, onde as aletas representam uma espécie de chicana. E nesse âmbito de estudo de trocadores de calor com canais aletados Karmo et al. [3] ressaltam que a transferência de calor por convecção nos trocadores é melhorada através da introdução de aletas nos canais do dispositivo. A configuração de trocador de calor aletado tem sido alvo de muitas investigações numéricas visando, em sua maioria, realizar uma alteração no parâmetro geométrico para maximização da transferência de calor, obtendo resultados significativamente melhores com essas configurações. Com ênfase semelhante, salienta-se também o trabalho de Sajedi et al. [4] que realizaram um estudo experimental e numérico para verificar a influência do número de aletas em um trocador de calor em relação à taxa máxima de transferência de calor e também a taxa de geração de entropia. Foi verificado que para as nove configurações de aletas testadas e para os diferentes números de Rayleigh avaliados a arquitetura do escoamento influencia consideravelmente no desempenho desse tipo de trocador de calor e ainda conseguiram estabelecer uma correlação entre a geometria e a taxa de transferência de calor.

Esse tipo de avaliação geométrica pode ser observada em vários trabalhos da literatura, onde destaca-se a utilização do Método Design Construtal em problemas de transferência de calor, que em maior parte, avaliaram e otimizaram vários Graus de Liberdade (GL) em cavidades das mais variadas formas geométricas inseridas em sólidos com geração de calor, objetivando maximizar a performance global de troca térmica, e.g. [5, 6, 7, 8, 9,10].

O Método Design Construtal, desenvolvido por Adrian Bejan, visa uma melhor distribuição das imperfeições através do emprego do método de restrições e objetivos, partindo do princípio físico que para que um sistema de fluxo de tamanho finito persistir no tempo (sobreviver) este deve evoluir de tal forma que forneça o mais fácil acesso às suas correntes de escoamento. Diversos exemplos de aplicação na engenharia, natureza e outras áreas podem ser vistos em [11, $12,13]$.

Diante do exposto, o presente trabalho utiliza-se do Design Construtal para avaliar a influência da geometria da aleta retangular a montante do escoamento $\left(H_{1} / L_{1}\right)$, da geometria da aleta retangular que está à jusante do escoamento $\left(H_{2} / L_{2}\right)$ e do distanciamento entre os centros das aletas $\left(L_{3}\right)$ sobre a taxa de transferência de calor entre as aletas e o escoamento circundante, que foi considerado com convecção mista, laminar e no regime permanente. Também objetiva-se investigar a influência do número de Grashof $\left(G r_{\mathrm{H}}\right)$ sobre o comportamento fluidodinâmico e térmico dos escoamentos analisados.

Essa análise parte da obtenção dos campos de temperaturas e do cálculo da taxa de transferência de calor por unidade de comprimento $q^{\prime}$ (W/m) através da solução das equações de conservação de massa, quantidade de movimento e energia com o Método de Volumes Finitos (MVF). Esse método numérico, de forma resumida, soluciona equações diferenciais parciais dividindo o domínio em pequenos volumes solucionando as equações de conservação em cada um desses volumes e integrando as soluções para obter uma solução para todo o domínio $[14,15]$. A metodologia utilizada também é recomendada em outros trabalhos onde o método Design Construtal é aplicado na avaliação geométrica de problemas com transferência de calor $[5,16,17$, $18,19]$. 


\section{MODELAGEM MATEMÁTICA E NUMÉRICA}

Para a análise do problema de transferência de calor por convecção mista para escoamentos incompressíveis, bidimensionais e no regime permanente resolvem-se as equações de conservação de massa, quantidade de movimento nas direções $x$ e $y$ e energia, respectivamente, dadas por [1]:

$$
\begin{aligned}
& \frac{\partial u}{\partial x}+\frac{\partial v}{\partial y}=0 \\
& \rho\left(u \frac{\partial u}{\partial x}+\frac{\partial u}{\partial y}\right)+\frac{\partial P}{\partial x}-\mu\left(\frac{\partial^{2} u}{\partial^{2} x^{2}}+\frac{\partial^{2} u}{\partial y^{2}}\right)=0 \\
& \rho\left(u \frac{\partial v}{\partial x}+v \frac{\partial v}{\partial y}\right)+\frac{\partial P}{\partial y}-\mu\left(\frac{\partial^{2} v}{\partial x^{2}}+\frac{\partial^{2} v}{\partial y^{2}}\right)+\rho g \beta\left(T-T_{\infty}\right)=0 \\
& \rho C_{p}\left(u \frac{\partial T}{\partial x}+v \frac{\partial T}{\partial y}\right)-k\left(\frac{\partial^{2} T}{\partial x^{2}}+\frac{\partial^{2} T}{\partial y^{2}}\right)=0
\end{aligned}
$$

onde $x$ e $y$ são as coordenadas espaciais (m), $u$ e $v$ são as componentes da velocidade nas direções $x$ e $y(\mathrm{~m} / \mathrm{s}), \rho$ é a massa especifica do fluido $\left(\mathrm{kg} / \mathrm{m}^{3}\right), P$ é a pressão estática do fluido $\left(\mathrm{N} / \mathrm{m}^{2}\right), \mu$ é a viscosidade dinâmica do fluido $\left(\mathrm{kg} / \mathrm{m}\right.$. s), $g$ é aceleração da gravidade na direção $y\left(\mathrm{~m} / \mathrm{s}^{2}\right)$ e $\beta$ é o coeficiente de expansão térmica $(1 / \mathrm{K}), T_{w}$ é a temperatura $(\mathrm{K})$ e o $T_{\infty}$ é a temperatura de referência $(\mathrm{K}), C_{p}$ é o calor específico à pressão constante $(\mathrm{J} / \mathrm{kg}$. K), e $k$ é a condutividade térmica do fluido (W/m. K). Vale destacar que, para a modelagem dos escoamentos com convecção mista, as forças de campo são consideradas através da hipótese de Boussinesq.

Para a solução das Eq. (1) - (4) utilizou-se o software comercial de dinâmica dos fluidos computacional (CFD - do inglês: Computational Fluid Dynamics) que é baseado no método de volumes finitos, denominado FLUENT ${ }^{\circledR}$. Esse código foi verificado através da solução de um problema semelhante proposto por Amaral Junior [20]. O solver utilizado é baseado na pressão, o acoplamento pressão-velocidade foi realizado com o uso do algoritmo SIMPLEC e para o tratamento dos termos advectivos foi empregado o esquema de interpolação Upwind de segunda ordem para as equações de conservação de quantidade de movimento e energia. Além disso, os cálculos foram considerados convergidos quando os resíduos para as equações de conservação de massa, quantidade de movimento e energia fossem menores do que $10^{-6}, 10^{-7}$ e $10^{-8}$, respectivamente.

As simulações numéricas foram realizadas usando um computador com processador Intel i5 com $2.2 \mathrm{GHz}$ de clock e $8 \mathrm{~GB}$ de memória RAM. O tempo de processamento para cada simulação foi em média de aproximadamente $1,20 \times 10^{2} \mathrm{~s}$.

$\mathrm{O}$ estudo de independência de malha do domínio computacional empregado investigou as malhas com os seguintes números de elementos triangulares: 5.558, 9.952, 13.952, 17.332, 22.180 e 28.776 elementos. Através da utilização do critério de $\left|\left(T_{\max }^{j}-T^{j+1}{ }_{\max }\right) / T_{\max }\right|<5,0 \times 10^{-4}$ obteve-se independência na malha com 22.180 elementos.

O problema a ser analisado é um escoamento laminar, incompressível, permanente, com convecção mista em um canal bidimensional com duas aletas com temperaturas prescritas altas em seu interior, ou seja, as aletas possuem temperatura $T_{\mathrm{W}}=330 \mathrm{~K}$. Na entrada do domínio serão impostos perfis de velocidade e temperatura constantes. A velocidade imposta é dada por $u_{\infty}$ de modo que o número de Reynolds do escoamento seja $R e_{H}=100$. Para a temperatura é imposto um valor inferior ao das superfícies aletadas de $T_{\infty}=310 \mathrm{~K}$. Já para as superfícies inferior e superior (região hachurada da Fig.1) é imposta uma condição térmica adiabática. Do ponto de vista fluidodinâmico, tem-se uma condição de não deslizamento e impermeabilidade nas superfícies hachuradas (Fig. 1) e nas aletas. Na saída do canal, aplica-se a condição de pressão manométrica 
nula para o campo fluidodinâmico e fluxo nulo para o campo térmico. O domínio computacional possui as seguintes dimensões: $H=50 \mathrm{~mm}, L=800 \mathrm{~mm}$ e $L_{e}=500 \mathrm{~mm}$.

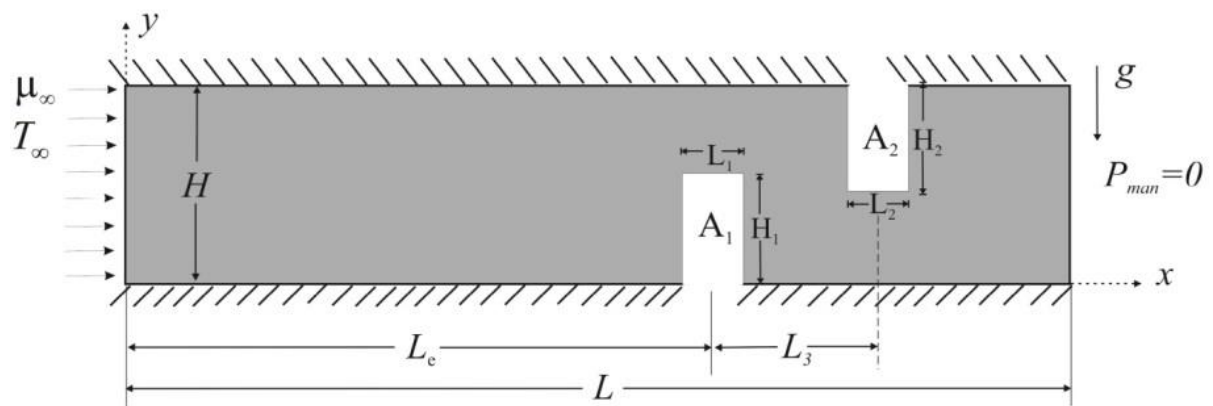

Figura 1: Domínio Computacional do problema.

Define-se o fluido do escoamento, a partir do número de Prandtl, um número adimensional definido por $\operatorname{Pr}=v / \alpha$, onde neste trabalho é assumido $\operatorname{Pr}=0,71$. Quanto aos escoamentos analisados, os mesmos também são caracterizados por dois adimensionais: o primeiro é o número de Reynolds, que é dado por $R e_{H}=\rho v H / \mu$, que foi considerado igual a 100 para todos os escoamentos, e o segundo é o número de Grashof, que é definido por $G r_{H}=g \beta\left(T-T_{\infty}\right) H^{3} / v^{2}$. Para as simulações foram considerados para o número de Grashof, os valores de: $10^{3}, 10^{4}$ e $10^{5}$.

Quanto à geometria, considerou-se a restrição da área do canal $(A=H . L)$, como sendo 40.000 $\mathrm{mm}^{2}$. A área das aletas $\left(A_{1}=H_{1} \cdot L_{1}\right.$ e $\left.A_{2}=H_{2} . L_{2}\right)$ são mantidas fixas, além de, no presente estudo, serem consideradas iguais entre si. Essa condição de igualdade é uma opção da pesquisa, visto que estas áreas poderiam ser distintas uma da outra. Com essa restrição para as áreas das aletas, tem-se também uma restrição da ocupação das aletas em relação à área total do canal, definida por $\phi_{1}=\phi_{2}=0,0125$. A partir dessas restrições, busca-se uma geometria otimizada na razão para as dimensões da aleta a montante $\left(H_{1} / L_{l}\right)$ e também para a razão da aleta a jusante do escoamento $\left(H_{2} / L_{2}\right)$, e ainda o distanciamento entre os centros das aletas $\left(L_{3}\right)$ para que se obtenha a maximização da taxa de transferência de calor por unidade de comprimento, que é dado por $q^{\text {' }}$ $(\mathrm{W} / \mathrm{m})$, do fluido com as aletas para um escoamento em regime permanente.

Mais precisamente, neste estudo a razão $H_{l} / L_{l}$ foi variada na faixa entre $0,25 \leq H_{l} / L_{l} \leq 4,0$ com um incremento de 0,25 , e de modo idêntico, foi variada a razão $H_{2} / L_{2}$. E ainda, para o último GL foi variado para $L_{3}=50,0 \mathrm{~mm}, 100,0 \mathrm{~mm}$ e $200,0 \mathrm{~mm}$. Neste processo manteve-se fixo um valor de $L_{3}$ e para cada valor de $H_{2} / L_{2}$ variaram-se as 16 possibilidades de $H_{1} / L_{1}$. O processo é repetido para os demais valores de $L_{3}$ já mencionados. Essa variação tem o intuito de buscar a geometria que leva a um valor máximo para o $q^{\prime}$, processo que pode ser observado na Fig. 2.

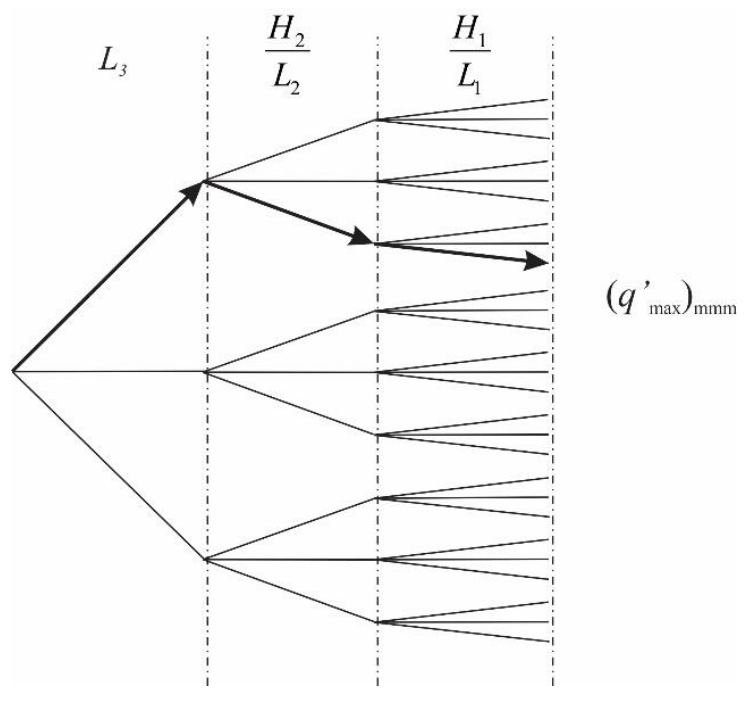

Figura 2: Esquema ilustrando o processo de otimização realizado aplicando o método Design Construtal com mecanismo de busca exaustiva. 


\section{RESULTADOS E DISCUSSÃO}

Analisando os escoamentos para $R e_{H}=100$ e $\mathrm{Gr}_{H}=10^{3}$, percebe-se uma predominância da convecção forçada, que pode ser observada através do o número de Richardson, dado por $R i=$ $G r / R e^{2}$, que nesse caso é igual a 0,1 , onde há predominância da convecção forçada. Partindo desses parâmetros, mostra-se inicialmente o efeito da razão da aleta a montante $\left(H_{1} / L_{1}\right)$ para várias magnitudes da razão $H_{2} / L_{2}$ sobre a taxa de transferência de calor $q^{\prime}(\mathrm{W} / \mathrm{m})$. Pode-se perceber que para o $G r_{H}=10^{3}$ os melhores resultados foram obtidos quando essa razão teve sua altura máxima para os casos analisados, ou seja, $H_{l} / L_{l}=4.0$. Quanto ao distanciamento, quando $L_{3}=50 \mathrm{~mm}$ obteve-se os máximos globais, como ilustra a Fig. 3(a). Ressalta-se que para $L_{3}=100$ mm e 200 mm obteve-se comportamentos semelhantes para ambos, então na Fig. 3(b) apresentase as curvas de efeito para apenas para quando $L_{3}=100 \mathrm{~mm}$. Além disso, destaca-se que quando $H_{l} / L_{l}=0.25$ obteve-se um ponto de máximo local para $q^{\prime}(\mathrm{W} / \mathrm{m})$ nas menores razões.

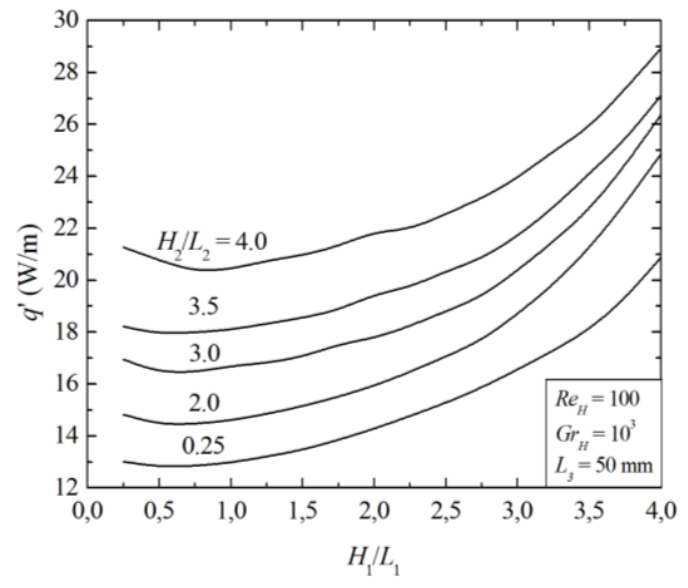

a)

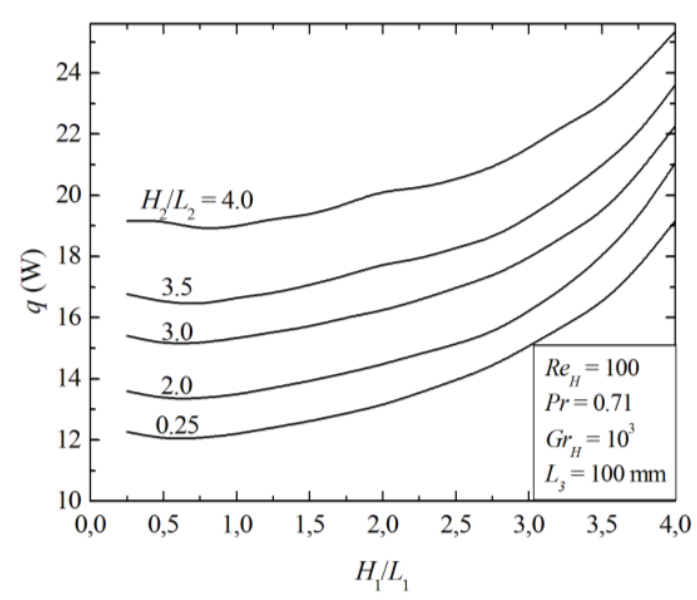

b)

Figura 3: Efeito da razão $H_{1} / L_{1}$ sobre o q' $(W)$ para várias razões $H_{2} / L_{2}, G r_{H}=10^{3}$ e diferentes distâncias entre aletas: a) $L_{3}=50 \mathrm{~mm}$, b) $L_{3}=100 \mathrm{~mm}$.

A partir dos resultados de $q^{\prime}{ }_{m}$, que é a taxa de transferência de calor uma vez maximizada, e de $\left(H_{1} / L_{1}\right)_{\mathrm{o}}$, que é denotado como a razão da aleta a montante uma vez otimizada, obtidos na Fig. 3 se constrói a Fig. 4, onde mais precisamente é mostrado o efeito da razão $H_{2} / L_{2}$ sobre o $q{ }^{\prime} m$ para os três diferentes valores de $L_{3}$ estudados aqui. Esses resultados evidenciam uma visível influência do GL do distanciamento entre os centros das aletas $\left(L_{3}\right)$, visto que quanto menor essa distância obteve-se a taxa de transferência de calor duas vezes maximizada, dada por $q^{\prime}{ }^{\prime}{ }_{m}(\mathrm{~W} / \mathrm{m})$. Da mesma forma que observado para o efeito da razão $H_{1} / L_{1}$, para $H_{2} / L_{2}$ o melhor desempenho foi obtido para a máxima magnitude desta razão, ou seja, $\left(H_{2} / L_{2}\right)_{\mathrm{o}}=4,0$. Como $\left(H_{1} / L_{1}\right)_{\mathrm{o}}=4.0$ para todas as situações, como mostra a Fig. 3, tem-se um comportamento constante e com isso, não se mostra relevante ilustrar o comportamento de $H_{2} / L_{2}$ sobre $\left(H_{1} / L_{1}\right)_{\mathrm{o}}$ para os três diferentes valores de $L_{3}$.

Deve ser destacado que o método Design Construtal permitiu uma melhoria significativa no desempenho térmico dos sistemas aletados. Por exemplo, para $\left(L_{3}\right)_{\mathrm{o}}=50 \mathrm{~mm}$ e $\left(H_{2} / L_{2}\right)_{\mathrm{oo}}=4,0$ foi observada uma diferença de $135 \%$ entre a geometria de $\left(H_{l} / L_{l}\right)_{\text {ooo }}=4.0$, e a pior geometria, quando $L_{3}=200 \mathrm{~mm}, H_{2} / L_{2}=0,25$ e $H_{1} / L_{1}=0,75$. 


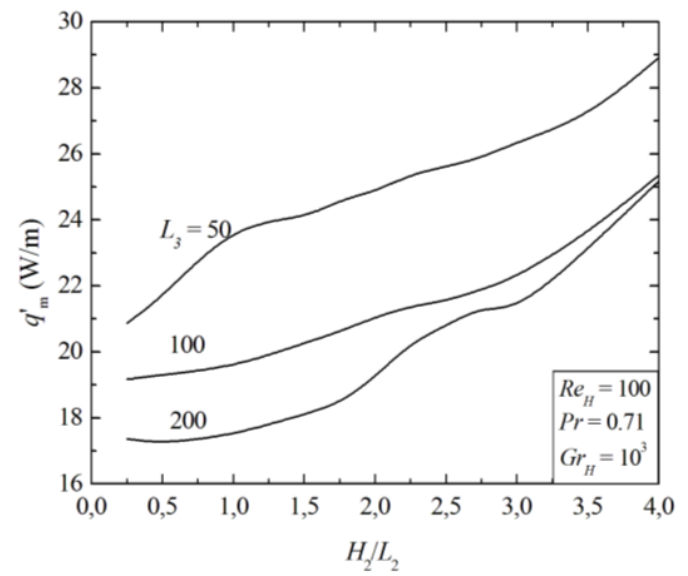

Figura 4: Efeito da razão $\mathrm{H}_{2} / \mathrm{L}_{2}$ sobre o q' ${ }_{m}$ para $\mathrm{Re}_{H}=100$ e $\mathrm{Gr}_{H}=10^{3}$.

Quanto ao comportamento térmico, mostra-se a distribuição dos campos de temperatura para as configurações geométricas que geraram os $q^{\prime}{ }_{m}$ para os três valores de $L_{3}$. Inicia-se com a Fig. 5(a), onde tem-se $H_{1} / L_{1}=H_{2} / L_{2}=4,0$ e $L_{3}=50 \mathrm{~mm}$, configuração geométrica que gera uma obstrução da passagem do fluido pelas aletas, causando a criação de um vórtice entre as mesmas. $\mathrm{Na}$ avaliação da Fig. 5 (b) onde as geometrias das aletas são iguais a da Fig. 5(a), porém com $L_{3}=$ $100 \mathrm{~mm}$, tem-se uma relativa diferença nos campos de temperatura, visto que esse maior distanciamento gerou um resfriamento do fluido entre as aletas e também uma menor intensidade da temperatura a jusante da segunda aleta até a saída do canal quando comparado com a Fig. 5 (a). E ainda, na Fig. 5 (c), onde se tem $L_{3}=200 \mathrm{~mm}$, o resfriamento entre as aletas cresceu consideravelmente em intensidade, e apenas próximo as paredes das aletas ocorre o aquecimento intenso.

(a) $L_{3}=50 \mathrm{~mm} \mathrm{e} H_{1} / L_{1}=H_{2} / L_{2}=4,0$.

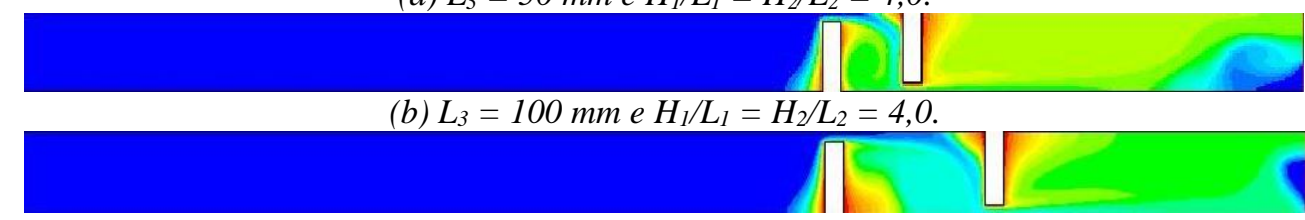

(c) $L_{3}=200 \mathrm{~mm}$ e $H_{1} / L_{1}=H_{2} / L_{2}=4,0$.
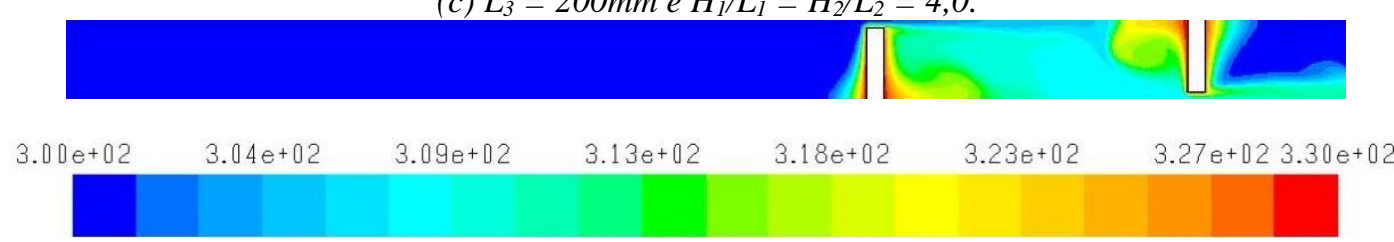

Figura 5: Distribuição dos campos de temperatura para um escoamento com $\operatorname{Re}_{H}=100$ e $\mathrm{Gr}_{H}=10^{3}:$ a) $\mathrm{L}_{3}$ $\left.=50 \mathrm{~mm}, b) L_{3}=100 \mathrm{~mm}, c\right) L_{3}=200 \mathrm{~mm}$.

O mesmo estudo desenvolvido para o escoamento com $R e_{H}=100$ e $G r_{H}=10^{3}$ foi repetido para o número de $G r_{H}=10^{4}$. A Figura 6, mostra o efeito da razão $H_{1} / L_{1}$ para várias razões $H_{2} / L_{2}$ sobre a taxa de transferência de calor $q^{\prime}(\mathrm{W} / \mathrm{m})$. Os resultados evidenciam que o máximo local ocorre para as menores razões, quando $H_{l} / L_{l}=0,25$, e tem-se um mínimo global quando $H_{l} / L_{l}=$ 0,75 e um máximo global novamente na maior magnitude estudada $\left(H_{l} / L_{l}=4,0\right)$.

Na Figura 7, realiza-se uma análise semelhante à realizada anteriormente, onde mostra-se o efeito da razão $H_{2} / L_{2}$ sobre o $q^{\prime}{ }_{m}$ para os diferentes distanciamentos $L_{3}$ analisados, o qual se evidencia a convergência para a geometria ótima das aletas quando $H_{1} / L_{1}=H_{2} / L_{2}=4,0$ e também o mesmo acontece para o terceiro GL, como mostra a Fig. 7. Observou-se também uma significativa diferença $(146 \%)$ na taxa de transferência de calor por convecção $\left(q^{\prime}\right)$ entre a melhor e pior configuração geométrica para $R e_{H}=100$ e $G r_{H}=10^{4}$. 


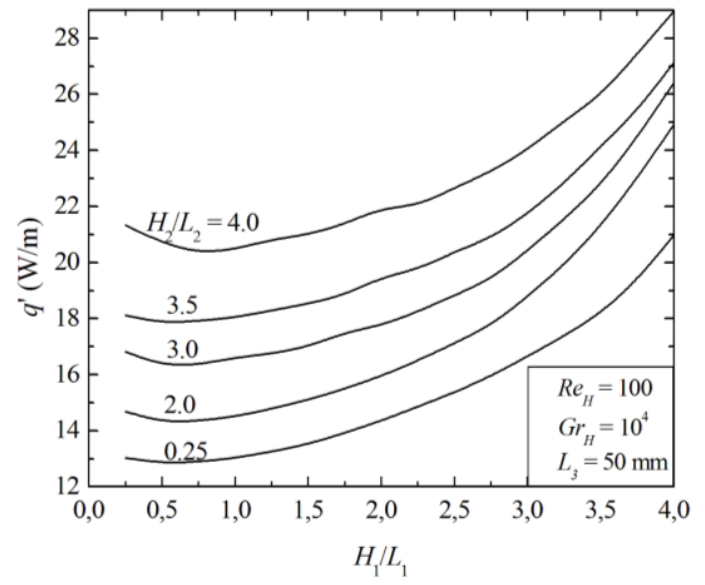

Figura 6: Efeito da razão $H_{1} / L_{1}$ sobre o q'para $\operatorname{Re}_{H}=100$ e $G r_{H}=10^{4}$.

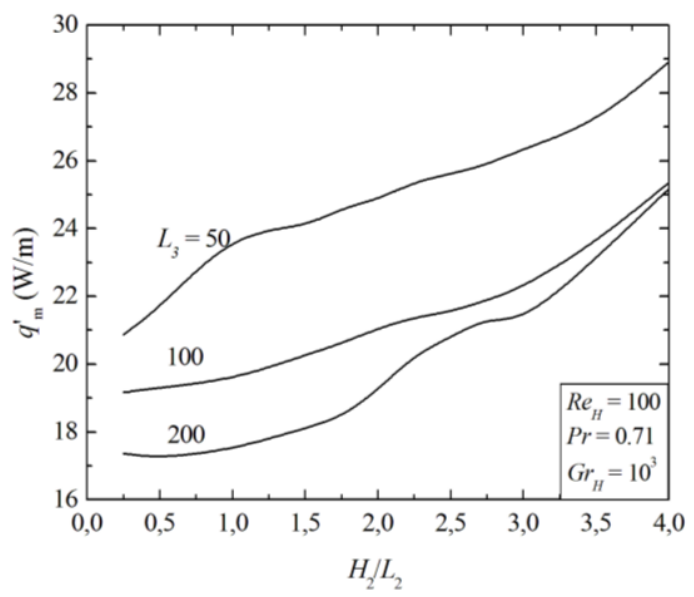

Figura 7: Efeito da distância $L_{3}$ sobre o $q_{m}^{\prime}$ para $\operatorname{Re}_{H}=100$ e $\mathrm{Gr}_{H}=10^{4}$.

Posteriormente, é apresentada a análise realizada para as simulações de escoamentos com $\operatorname{Re}_{H}$ $=100$ e $G r_{H}=10^{5}$, onde as Figs. 8 e 9 ressaltam novamente que há uma geometria ótima recomendada tanto para as dimensões das aletas $\left(H_{1} / L_{1}=H_{2} / L_{2}=4,0\right)$ quanto para $\mathrm{o}$ distanciamento entre os centros das aletas $\left(L_{3}=50 \mathrm{~mm}\right)$.

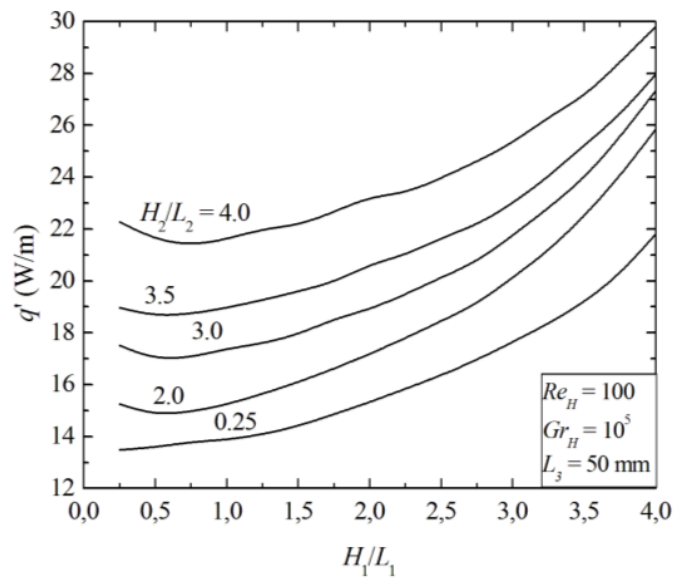

Figura 8: Efeito da razão $H_{1} / L_{1}$ sobre o q'em um escoamento com $\operatorname{Re}_{H}=100$ e $G r_{H}=10^{5}$ para $L_{3}=50$ mm. 


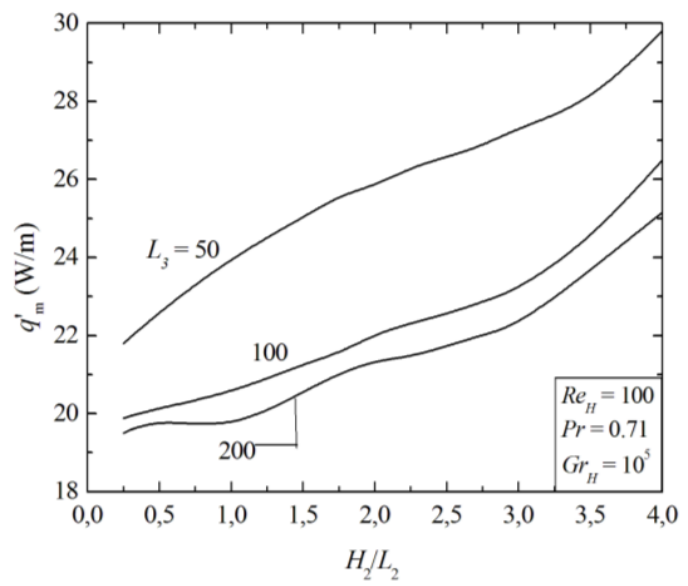

Figura 9: Efeito da razão $\mathrm{H}_{2} / \mathrm{L}_{2}$ sobre o q' ${ }_{m}$ para $\mathrm{Re}_{H}=100$ e $\mathrm{Gr}_{H}=10^{5}$.

Apesar de apresentarem uma mesma recomendação para as geometrias ótimas, os campos de temperatura mostram que com esses parâmetros de escoamento $\left(R e_{\mathrm{H}}=100\right.$ e $\left.G r_{\mathrm{H}}=10^{5}\right)$ há uma influência das forças de empuxo, devido ao número de Grashof simulado ter sido aumentado para $10^{5} \mathrm{e}$ assim, ter-se um número de Richardson $\left(R i=G r_{\mathrm{H}} / R e_{\mathrm{H}}{ }^{2}=10\right)$ que indica uma dominância da convecção natural no escoamento com convecção mista. Percebe-se na Fig. 10(a) que há um maior movimento ascendente do fluido próximo as aletas, causado pela influência das forças de empuxo e uma maior intensidade da temperatura do fluido na parede da aleta a jusante do escoamento, além de se notar que próximo a parte inferior do canal a intensidade dos campos é menor, observando-se assim diferenças significativas se comparado a Fig. 5 (a). Já para as Figs. 10(b) e (c), como as dimensões das aletas não obstruem o movimento do fluido, consegue-se perceber um comportamento característico laminar, com novamente uma influência das forças de empuxo, quando comparado com as Figs. 5 (b) e (c).

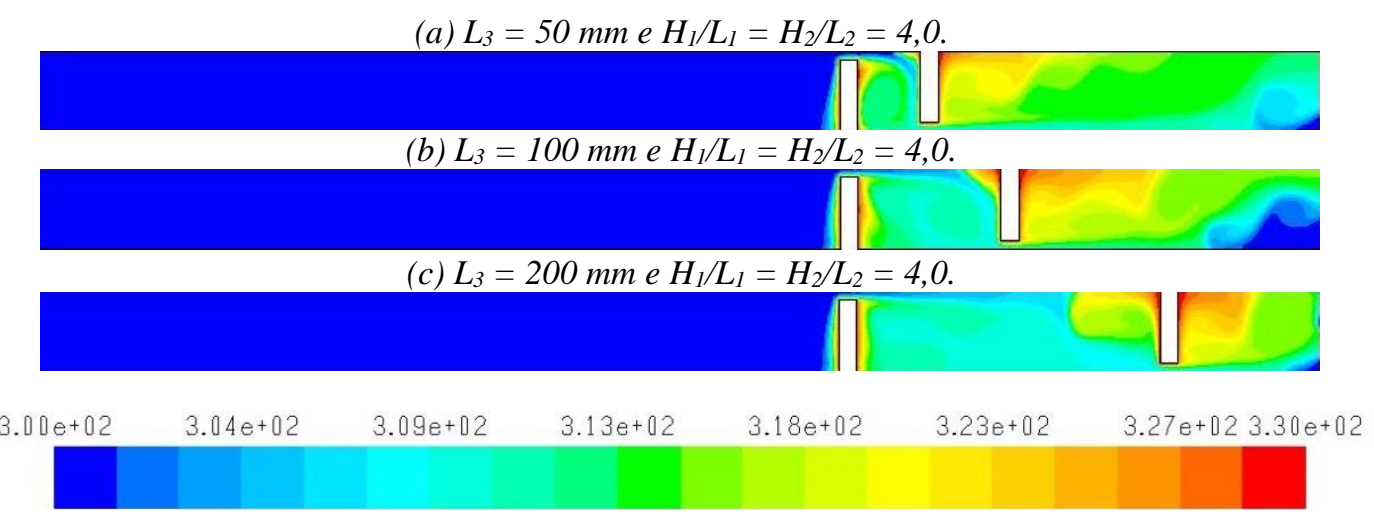

Figura 10: Distribuição dos campos de temperatura para um escoamento com $\operatorname{Re}_{H}=100$ e $G_{H}=10^{5}:$ a) $L_{3}=50 \mathrm{~mm}$, b) $L_{3}=100 \mathrm{~mm}$, c) $L_{3}=200 \mathrm{~mm}$.

Diante dessa análise, consegue-se mostrar a convergência para uma configuração ótima única, independente do número de Grashof simulado, tendo assim a razão da aleta a montante três vezes otimizada $\left.\left(H_{1} / L_{l}\right)_{\mathrm{ooo}}=4.0\right)$, a razão da aleta a jusante duas vezes otimizada $\left(H_{2} / L_{2}\right)_{\mathrm{oo}}$ e o distanciamento entre os centros das aletas uma vez otimizado $\left(L_{3}\right)_{\mathrm{o}}$. Observa-se um aumento de $q^{\prime}{ }_{m m m}$ em função do número de Grashof, o que era esperado, visto que o mecanismo motriz é intensificado, como nota-se na Fig. 11. 


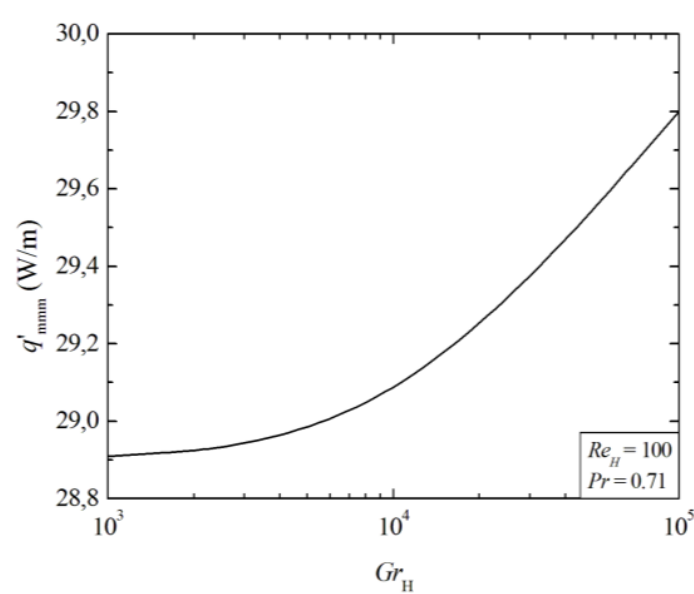

a)

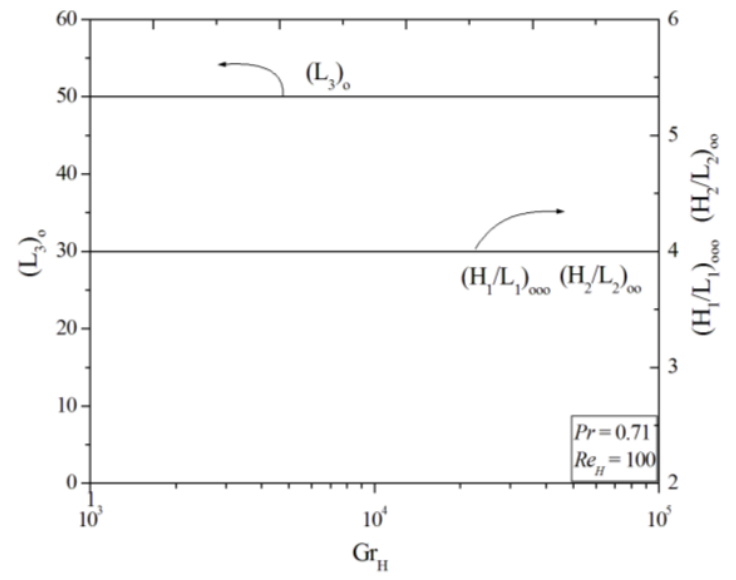

b)

Figura 11: Efeito do número de Gr sobre: a) q' ${ }^{\prime}{ }_{m m}$, b) optimal ratios $\left(L_{3}\right)_{o},\left(H_{2} / L_{2}\right)_{o o}$ e $\left(H_{3} / L_{3}\right)_{o o o}$.

\section{CONCLUSÃO}

Neste trabalho foram investigadas numericamente configurações geométricas de um canal aletado, com duas aletas, submetido a escoamentos laminares com convecção mista em regime permanente empregando o método de Design Construtal. Nesse contexto, realizaram-se simulações para escoamentos com números de Reynolds e Prandtl de $\operatorname{Re}_{H}=100$ e $P r=0.71$, avaliando-se três diferentes números de Grashof: $G r_{H}=10^{3}, 10^{4}$ e $10^{5}$. O Método dos Volumes Finitos foi empregado para resolver numericamente as equações de conservação de massa, de quantidade de movimento e energia. O principal objetivo foi avaliar a influência da geometria do sistema aletado $\left(H_{1} / L_{1}, H_{2} / L_{2}, L_{3}\right)$ a taxa de transferência de calor por convecção $\left(q^{\prime}\right)$ e também o efeito do $\mathrm{Gr}_{H}$ sobre a geometria através do Design Construtal.

Os resultados mostraram uma recomendação específica de geometria ótima para os casos estudados, obtendo para ambas as razões $\left(H_{1} / L_{1}\right)_{\mathrm{ooo}}=\left(H_{2} / L_{2}\right)_{\mathrm{oo}}=4.0$ (ou seja, para aletas com grande inserção no canal) e ainda para o distanciamento entre os centros delas: $\left(L_{3}\right)_{0}=50 \mathrm{~mm}$. Quanto ao comportamento da taxa de transferência de calor $\left(q^{\prime}\right)$, houve um aumento de mais de $100 \%$ da melhor para a pior configuração geométrica em uma mesma faixa de Grashof, ou seja, um grande ganho com um mesmo mecanismo motriz. Para a avaliação de $q^{\prime}{ }_{m m m}$ percebeu-se que um aumento de Grashof de $G r_{H}=10^{3}$ para $G r_{H}=10^{4}$ não ocorreram relevantes mudanças no $q^{\prime}{ }_{m m m}$ e consequentemente não houve mudanças significativas nas distribuições dos campos de temperatura. Em função da geometria ótima ter sido avaliada apenas sob o enfoque térmico, torna-se relevante em futuros trabalhos investigar a influência das razões geométricas estudadas na perda de carga do escoamento, considerando um problema multiobjetivo.

\section{AGRADECIMENTOS}

Os autores M. V. Altnetter e M. dos S. Pereira agradecem a CAPES pela bolsa de estudos e os autores L. A. Isoldi, L. A. O. Rocha e E. D. dos Santos agradecem ao CNPq pela bolsa de produtividade em pesquisa.

\section{REFERÊNCIAS BIBLIOGRÁFICAS}

1. Bejan A. Convection Heat Transfer. Durham: John Wiley; 2004. 696 p.

2. Siegel R., Howell JR. Thermal Radiation Heat Transfer. New York: Taylor \& Francis, 4th edition; 2002. $372 \mathrm{p}$.

3. Karmo D, Ajib S, Khateeb AA. New method for designing an effective finned heat exchanger. Appl Therm Eng. 2013;(51):539-50, doi: 10.1016/j.applthermaleng.2012.09.042. 
4. Sajedi R, Taghilou M, Jafari M. Experimental and numerical study on the optimal fin numbering in an external extended finned tube heat exchanger. Appl Therm Eng. 2015;(83):139-46, doi: 10.1016/j.applthermaleng.2014.12.040.

5. Bejan A, Almogbel M. Constructal T-shaped fins. Int J Heat Mass Transfer. 2000;(43):2101-15, doi: 10.1016/S0017-9310(99)00283-5.

6. Biserni C, Rocha LAO, Stanescu G, Lorenzini E. Constructal H-shaped cavities according to Bejan's theory. Int J Heat Mass Transfer. 2007;(50):2132-38, doi: 10.1016/j.ijheatmasstransfer.2006.11.006.

7. Aldrighi ES, Rodrigues PM, Rodriguez BDA, Isoldi LA, Rocha LAO, Dos Santos ED. Constructal Design of Rectangular Fin Intruded into Different Surfaces of Forced Convective Lid-Driven Cavity Flow. Int J Fluid Mech Research. 2016;(43):418-40, doi: 10.1615/InterJFluidMechRes.v43.i5-6.50.

8. Lorenzini, G, Biserni C, Estrada ED, Isoldi LA, Dos Santos ED, Rocha LAO. Constructal Design of convective Y-Shaped cavities by means of genetic algorithm. J Heat Transfer. 2014;(136):071702-1-10, doi: 10.1115/1.4027195.

9. Lorenzini G, Biserni C, Rocha LAO. Geometric optimization of X-shaped cavities and pathways according to Bejan's theory: comparative analysis. Int J Heat Mass Transfer. 2014;(73):1-8, doi: 10.1016/j.ijheatmasstransfer.2014.01.055.

10. Lorenzini G, Rocha LAO. Geometric optimization of T-Y-shaped cavity according to constructal design. Int J Heat Mass Transfer. 2009;(52):4683-46, doi: 10.1016/j.ijheatmasstransfer.2009.06.020.

11. Bejan A, Lorente S. The Constructal Law (La Loi Constructale). Int J Heat Mass Transfer. 2006;(49):445-445, doi: 10.1016/j.ijheatmasstransfer.2005.07.031.

12. Bejan A, Lorente S. Design with Constructal Theory, New Jersey: John Wiley; 2008. 572 p.

13. Bejan A, Zane JP. Design in Nature: how the constructal law governs evolution in biology, physics, technology, and social organization, New York: Doubleday; 2012. 296 p.

14. Maliska CR. Transferência de Calor e Mecânica dos Fluidos Computacional. Rio de Janeiro: Livros Técnicos e Científicos Editora S.A.; 2004. 453 p.

15. Versteeg HK, Malalasekera W. An Introduction to Computational Fluid Dynamics: The Finite Volume Method, Pearson; 2007. 503 p.

16. Bejan A, Dan N. Constructal trees of convective fins. Int J Heat Mass Transfer 1999;(121):675-682, doi: 10.1115/1.2826032.

17. Biserni C, Rocha LAO, Bejan A. Inverted fins: geometric optimization of the intrusion into a conducting wall. Int J Heat Mass Transfer. 2004;(47):2577-86, doi: 10.1016/j.ijheatmasstransfer.2003.12.018.

18. Bejan A, Rocha LAO, Lorente S. Thermodynamic optimization of geometry: T- and Y-shaped constructs of fluid streams. Int J Therm Sci. 2000;(39):949-60, doi: 10.1016/S1290-0729(00)01176-5.

19. Lorenzini G, Biserni C, Corrêa RL, Dos Santos ED, Isoldi LA, Rocha LAO. Constructal design of Tshaped assemblies of fins cooling a cylindrical solid body. Int J Therm Sci. 2014;(83):96-103, doi: 10.1016/j.ijthermalsci.2014.04.011.

20. Amaral Junior JB. Convecção mista em escoamento laminar ou turbulento num canal aquecido inferiormente com fontes discretas, Itajubá, (MG): UNIFEI; 2007. 